\title{
Redirection of Client/Server Relationship of $X$ Window System as a Simple, Low-Cost, Departmental Picture Archiving and Communication System Solution for Nuclear Medicine
}

\author{
Frederick L. Datz, Donald A. Baune, and Paul E. Christian
}

\begin{abstract}
Picture archiving and communication systems (PACS) offer significant advantages over current film-management techniques. However, PACS are complex and expensive, factors that have limited their entry into the radiology and nuclear medicine communities. We present a simple, low-cost PACS solution that allows viewing of images from different computer systems by redirection of the $X$ Window system. In this technique, multiple copius of the imaging software are remotely opened from generic UNIX workstations interfaced to the main computer system via Transmission Control Protocol/Internet Protocol over Ethernet. The $X$ Window system that provides the windowing system for the main computer is redirected to the workstations' displays. With this technique, viewing and processing of images on a remote station is virtually identical to working at the main computer's console. The technique requires that the commercial imaging system's hardware, operating system, and imaging software support multiuser multitasking and the execution of multiple copies of its imaging software, and that they use $X$ Windows as the graphical system. Advantages of the technique include low cost, ease of maintenance, ease of interconnecting different types of computers, the capacity to view images regardless of file format, and the capacity to both view and process images. The latter is a necessity for modalities such as nuclear medicine. A disadvantage of the technique is that the number of nodes that can be supported is limited.
\end{abstract}

Copyright 1994 by W.B. Saunders Company

KEY WORDS: picture archiving and communication systems, computers, nuclear medicine.

$\mathbf{P}$ ICTURE ARCHIVING and communication systems (PACS) apply digital technology to the problem of film storage and viewing. ${ }^{1}$ PACS offer significant advantages over the current practice of archiving images in file rooms and viewing films on light-boxes or alternators. Digital storage of images speeds access, prevents film loss and misplacement, and allows multiple physicians at different locations to view the same images simultaneously. ${ }^{1.2}$ The display of images on computer screens allows the adjustment of image brightness and contrast for optimal viewing. ${ }^{3}$

Unfortunately, PACS are complex and expensive, factors that have slowed implementation in radiology departments. ${ }^{4}$ For these reasons, many experts now recommend the progressive introduction of PACS into a radiology department one modality at a time rather than implementing a global PACS system. ${ }^{4}$ The local PACS systems can grow into a more widespread PACS system by interfacing the local networks. ${ }^{5}$ Complete digitization of a radiology department may require as long as 15 to 20 years. ${ }^{4}$ The method of progressive implementation of PACS is sometimes called the bottom-up approach.

At first glance, nuclear medicine seems to be a perfect modality for PACS implementation. Its output is digital, the relative low resolution of its images allows the use of less-expensive displays, and file storage needs are small compared with computed tomography (CT), magnetic resonance imaging, (MRI) and plain radiographs. ${ }^{6,7}$ However, unlike other radiologic modalities in which acquired images are viewed statically without processing, nuclear medicine requires dynamic image displays and the ability to process studies. In addition, nonimage data such as time-activity curves are generated.

A number of nuclear medicine examinations require cinematic display. Cardiac-gated bloodpool acquisitions (MUGA's), gated singlephoton emission computed tomography (SPECT), gastrointestinal bleeding studies, and biliary scans are all viewed with a closed loop movie or cine display. Three-dimensional (3D) reconstruction of images with surface rendering, a technique that is becoming more common in nuclear medicine, requires a continuously rotating display, as well. ${ }^{8}$

Secondly, many nuclear medicine studies are

From the Division of Nuclear Medicine and Medical Imaging Research Laboratory, Department of Radiology, University of Utah School of Medicine, Salt Lake City, Utah.

Address reprint requests to Frederick L. Datz, MD, Director, Division of Nuclear Medicine, Department of Radiology, Room 1B-647, University of Utah School of Medicine, Salt Lake City, Utah 84132.

Copyright 1994 by W.B. Saunders Company 0897-1889/94/0703-0014\$3.00/0 
processed. All SPECT studies are reconstructed with significant operator interaction, including the use of real-time viewing of filter parameter choices. MUGA's and gated Tc-99m sestamibi studies must be processed to calculate the leftand right-ventricular ejection fractions, in addition to other parameters of cardiac function. This can be done automatically, but the operator has the option of changing parameters such as the background, or performing the operation manually. Cardiac computer-assisted diagnostic programs and phase-analysis software require operator interaction, as well..$^{9.10}$ Noncardiac studies such as renal scans, biliary ejection fractions, and gastric emptying must also be processed to quantitate physiologic parameters.

Finally, many studies such as renal scans include nonimage data such as time-activity curves. These complex viewing and processing requirements prevent generic PACS viewing software aimed at all radiologic modalities from being adequate for nuclear medicine.

Writing specific nuclear medicine PACS software that runs on remote systems and mimics the viewing and processing capabilities of the imaging software provided by the camera equipment manufacturer is very difficult and time consuming. For this reason, we have developed a PACS solution for nuclear medicine based on redirection of the $\mathrm{X}$ Window system from the main computer system to remote generic UNIX workstations that allows viewing of static and cine studies as well as processing of images. The technique allows easy connection of different types of computers and remote viewing of images regardless of their file format, yet is relatively low cost and easy to maintain.

We present our system below. Although this system was implemented for nuclear medicine, the technique can be applied to other modalities as well if the main computer system's hardware, operating system, and imaging software supports multiuser multitasking and the execution of multiple copies of its imaging software, and uses the $\mathrm{X}$ Window system as its graphics system. The capacity to process images may become important for CT and MRI as 3D reconstructions and $\mathrm{MR}$ angiography are perfected.

\section{MATERIALS AND METHODS}

\section{Hardware}

The main computer systems were two Stardent 750 computers (Kubota Pacific Computers, Inc) with a reduced instruction set computer (RISC) processor running 64 million instructions per second (MIPS), 96 MFLOPS, with 32 Mbyte of random access memory (RAM). and a Stardent 1500 computer with a RISC processor running 32 MIPS. 33 MFLOPS, with $32 \mathrm{MB}$ of RAM. The displays were $19 \mathrm{in}$ in size and 1,280 $\times 1,024$ pixels in resolution. Each system was networked to a Picker $750 \mathrm{MB}$ Winchester hard drive and four 2.1 GB Sun hard drives (Sun Microsystems Inc. Mountain View, CA) configured as a disk farm. Four Picker SPECT cameras (Picker International, Bedford Heights, $\mathrm{OH})$ were interfaced to the main computers.

Remote viewing stations were seven Sun LX workstations with a RISC processor running 59.1 MIPS, 4.6 MFLOPS, with $24 \mathrm{MB}$ of RAM, a GX+ graphics accelerator, and a local 424 Mbyte hard drive; one Sun SPARC 2 workstation with a RISC processor running 28.5 MIPS, 4.2 MFLOPS, with 16 Mbyte of RAM, and a local hard drive of 424 Mbyte: and one Sun IPX with a RISC processor running 28.5 MIPS, 4.2 MFLOPS, with 16 Mbyte of RAM and a local hard drive of 424 Mbyte. All of the LX workstations had an extra 1 Mbyte of video RAM to allow a screen resolution of $1,280 \times 1,024$ pixels; four of the systems had 19-in monitors, two had 16-in monitors. The SPARC 2 and the IPX systems had 16-in monitors with a resolution of $1,152 \times 900$ pixels.

The Stardents and the SPARC 2 were connected to the Ethernet backbone by transceivers; the LX's were connected to an Ethernet backbone via 10BaseT.

\section{Software}

General software. The Stardent computers used AT\& T UNIX system $V$, release 4 (Middletown, NJ) and the $X$ Window system version 11 , release 4 . The imaging software was Picker Odyssey Baseline 5. This combination allowed multiuser multitasking, and multiple copies of the imaging software to execute simultaneously. The LX computers used Solaris 2.1, Sun's operating system (SUNOS), which is AT\&T UNIX system $V$, release 4 compliant. The Sun SPARC 2 and the IPX used SUNOS 4.1. which is compliant with BSD UNIX, version 4.3 (Berkley Software Distribution, Berkley, CA). All systems used the OPENLOOK/ Open Windows graphical user interface (GUI), which is based on the X Window system.

All systems were networked via the Transmission Control Protocol/Internet Protocol (TCP/IP). The file system was networked via Network File System (Sun Microsystems Inc, Mountain View CA).

Redirection of the $X$ Window system. The redirection of the $\mathrm{X}$ Window system from the main computers was performed as follows. Shell scripts were written for each remote UNIX workstation and were placed in the login file of the C-shell so that the script was executed automatically during log-in each day. A shell script for the main computer system was also programmed. The script on each worksta- 
tion is shown below.

$$
\begin{aligned}
& \text { \#!/bin/csh } \\
& \text { xhost }+ \\
& \text { rsh prism doit }
\end{aligned}
$$

The first line forces the shell scrip to execute under the C-shell because by-default scripts are executed under the Bourne shell. The xhost + command allows any other computer on the network to display on the remote system. The command can atso be written so that only a specific computer is allowed to direct its graphic output to the remote system. $r s h$ opens a remote shell on one of the main computer systems, which was named prism. doit is a script that runs on the prism. The second script is as follows;

\#!/bin/csh
setenv DISPLAY nucmedI:0.0
xrdb-load Xdefaults
dials\&
workbench\&
freediskd

The setenv DISPLAY nucmed 1:0.0 Jine, under the C-shell, changes the global environmental variable of the main computer's display to that of the remote station's display, thereby redirecting the $X$ Window system to the remote system's display. The .Xdefaults line adds the values in the main computer's .Xdefault file to the remote system's $X$ environment. These could be permanently added to the remote computer's .Xdefaults file; however. having them read in with each log-in allows any modifications of the main computer system's Xdefaults files to be automatically added to the remote system's environment. The last three lines start the imaging software; the \& puts them in the background.

Because the $\mathrm{X}$ Window commands are sent from the main computer to the remote. the same fonts must be available on both systems. This required additional fonts to be loaded on the remote systems.

File storage software. Alt images were acquired onto a Picker 750 Mbyte hard drive. Immediately after acquisition. the images are pushed to the Sun disk farm. Here a daemon automatically changes file permissions so that only root has permission to delete files.

Other software. A TE320 terminal emulator was also loaded onto each remote viewing system. Through a separate window, the emulator allowed access to the radiology department's information system, IDXrad, which runs on a Digital Equipment Corporation VAX 9000 (Waltham, MA).

\section{Location of Systems}

The main computers were located in the imaging suites of the University Hospital Nuclear Medicine Department. Four of the remote systems were located in the nuclear medicine reading room, approximately $20 \mathrm{~m}$ from the imaging area. One LX and the SPARC 2 were located in faculty offices approximately $30 \mathrm{~m}$ from the imaging area.
One $\mathrm{LX}$ was placed in a conference room $60 \mathrm{~m}$ from the imaging area. Two LX's were placed in the main radiology reading room and the bone reading room approximately 200 $m$ from nuclear medicine. The IPX was located at the Veteran's Administration Hospital's Nuclear Medicine Department, approximately I mile from the University Hospital. That system was connected to the computers at the University Hospital via Ethernet over a microwave link.

\section{Tests of System Performance}

To determine what load the remote viewing stations could place on the main computer system without appreciably slowing access to images or crashing the system, a test was devised in which up to five LXs continuously accessed. manipulated, and processed images during image acquisition. Five LXs were networked to the slower main computer system, the Stardent 1500. The Stardent 1500 was acquiring a SPECT cardiac scan. Remote systems were brought on line one at a time until two LX's were continuously displaying cines, two LX's were accessing images and scrolling through image files, and one $L X$ was processing images. This test was considered more stressful than what would occur in clinical practice because processing or even viewing by more than three remote systems at any one time was felt to be unlikely.

In addition, the system was used for 3 months to determine its clinical efficacy. The nuclear medicine physicians using the system were then queried as to the system's performance compared with using the main computer's console. A grading system of 1 to 10 was used, with 10 equal to the main system's console. The overall reliability was also measured.

\section{RESULTS}

The system could support the simultaneous acquisition of a SPECT study and the manipulation of images on five remote viewing stations without any deleterious effect on image acquisition. However, the remote stations caused a gradual slowing of image viewing and processing after three systems came on line. Four on-line stations caused a mild slowing; five caused noticeable slowing, although not to a point that the remote systems were clinically unusable.

Three nuclear medicine physicians using the remote systems for clinical use graded the system at 9.1. The only disadvantage was the somewhat slower screen rewrite speed of the SPARC 2 and IPX compared with the main computer console.

The reliability of the system and time needed to administer it were equal to our Sun computer network's. There was no major down time for the system over the test period. 


\section{DISCUSSION}

The $\mathrm{X}$ Window system was developed at Massachusetts Institute of Technology (MIT) as part of project Athena. ${ }^{11.12}$ The goal of the project was to allow interactive computing on any of the campus's many different computer systems. The MIT program was based on a windowing system developed at Stanford University (Stanford, CA) named "W." The next letter in the alphabet, $\mathrm{X}$, was used to name the new MIT graphical system.

The $\mathrm{X}$ Window system became commercially available in 1986; version 11, release 5 was the most current update at the time this article was written. The $\mathrm{X}$ Window system is to UNIX computers what Microsoft Windows is to the IBM PC and IBM clones. However, unlike Microsoft Windows, $\mathrm{X}$ Window has become a standard that is not controlled by any one software or hardware company. This standardization has made $\mathrm{X}$ Window the primary graphical windowing system used on virtually all UNIX computers. There are two different GUI's or window managers that sit atop $\mathrm{X}$ Window, OPENLOOK and Motif. However, recent agreements between major equipment manufacturers will make Motif the standard GUI.

The $\mathrm{X}$ Window system is based on a client/ server architecture. ${ }^{11}$ Clients, or application programs, communicate with servers, or display units. This can occur within a stand-alone workstation, or the communication can be between two machines over a network. Client/server networks can be developed in which diskless terminals have their graphical displays controlled by a central computer system; these diskless terminals are called $\mathrm{X}$ terminals. However, this technique has never become popular.

The client/server relationship of the $\mathrm{X}$ Window system is different from the classical client/ server relationship in which a file server supplies data to a number of remote computers, or clients. "In the X Window system, the server is located on the user's computer or X terminal and the client is located on a remote, often larger computer system. The server passes user input to the attached client. User input includes mouse button clicks, key presses, and changes in pointer location. The client processes the user input and sends back $X$ Window commands that request the server to draw graphic objects. The server decodes the client messages and generates the appropriate graphical images.

The $\mathrm{X}$ Window system is not based on any particular computer hardware architecture. Our technique can display images on virtually any brand of UNIX workstation or X terminal. In theory, the X Window system will run on PC's and Macintoshs if the appropriate $\mathrm{X}$ emulation software is running locally. We have successfully sent images to a PC this way; however, we have not performed any detailed experiments.

Huang ${ }^{13}$ lists three methods for implementing a PACS system. ${ }^{13}$ In the first, system integration, the hospital assembles a multidisciplinary team that chooses PACS hardware components from a variety of manufacturers and writes software to integrate the system. In the second, the PACS system is designed by the hospital based on its unique needs; then a manufacturer is contracted to provide the system. The third method is to purchase a turnkey system.

The advantages of the first approach are that it can be tailored to the needs of the department and it can be continuously upgraded with stateof-the-art components, preventing obsolescence. The disadvantage is that a unique PACS system can be expensive to create, slow to implement (software development and testing can require several years), and difficult to maintain. The second of Huang's methods is expensive. The turnkey approach is somewhat less expensive than the second method, but by the time the turnkey system is available, some of the components may already have become obsolete.

Our approach to PACS is closest to Huang's first method, but is without many of the disadvantages. By using generic UNIX workstations and having image processing occur on a central computer system that is already in place for image acquisition, the price of the PACS system is kept reasonably low. Second, because the technique uses redirection of the $\mathrm{X}$ Window system to display the main computer's already existing imaging software, the PACS system can be implemented quickly. Finally, it is not difficult to maintain because any trained UNIX system administrator can oversee the system.

Our system has a number of advantages over a turnkey PACS system. First, it allows the use of existing equipment. We already owned the Stardent computers; we also had several work- 
stations that were being used for research. Second, it does not lock the user into a specific PACS manufacturer; in the future, we are free to switch to any brand of workstation we wish. Third, the upgrade path with UNIX workstations is much better than with most dedicated medical equipment. Sun, for example, allows trade-ins and upgrades of existing equipment that can result in significant savings. A fourth advantage is ease of use. By redirecting the $\mathrm{X}$ Window system, we can use the same imaging software with which we are already familiar. The software is powerful; it is not a strippeddown version of our regular imaging software. Finally, our solution allows both viewing and processing of images on remote systems. The latter is usually not possible with commercial PACS systems.

Another advantage of our system is that images can be remotely displayed on different computers regardless of the original image file format. Standards for radiologic image files are currently being developed in the United States by the American College of Radiology and the National Electrical Manufacturers Association; in Europe, the Interfile standard is being proposed for nuclear medicine. ${ }^{7}$ However, a standard file format that is usable by most radiologic modalities will be difficult to apply to nuclear medicine. For example, new techniques for SPECT imaging require that gantry position information be placed in the file in addition to the image data. ${ }^{14}$ Such needs are not adequately addressed in any currently proposed image file format standard.

We have found other benefits of our PACS approach. Because the UNIX workstations are not dedicated viewing stations, they can be used for other tasks such as scientific computing. Because the workstations can multitask, we can run research computing jobs in the background. In addition we use the workstations for word processing, slide making, teaching (using a digital teaching file), and video conferencing (with the addition of a TV camera and frame-capture board).

There are disadvantages to our approach. Because we are using the same imaging software that was designed for nuclear medicine specialists, the interface can be confusing for radiologists in other subspecialties. However, we found that approximately a half hour of training allowed them to access images without difficulty. The problem of complexity of use would be even worse for clinicians; however, the system is intended for use by our own radiology department personnel.

An important problem with allowing remote access to the fully functioning imaging software is the possibility of accidental (or even purposeful) deletion of patient files. It is for this reason that all acquisitions are immediately pushed to the disk farm. Here, a daemon automatically changes file permission so that no one other than root can delete files.

Heavy use of the remote workstations can slow the main computer system; however, in actual practice, we have not found this to be a significant problem. However, if more workstations were networked into the system, significant slowdowns could occur. Based on current use, we estimate that 15 workstations could be supported ( 5 on each main computer system) without a significant effect on system performance on the same Ethernet segment or on segments separated by a bridge or other device. Note that this is based on our hardware and the workload in our nuclear medicine division; the exact number of supportable nodes will vary depending on these variables.

A related problem is the effect of increased network traffic generated by redirection of the $\mathrm{X}$ Window system on overall network performance. Each time a user clicks the mouse or moves the cursor, the remote system must send a message to the central computer system. The central system then responds with an $\mathrm{X}$ command that the remote system performs. This is similar to networked diskless systems. The increased number of packets sent over the Ethernet can cause numerous collisions that will slow network traffic for all users. This has not occurred with our system, primarily because of the significant amount of time in which the workstations are idle. Once a patient image is accessed and processed, it can remain on screen without any further interaction with the main computer system. If heavy use affects network traffic speed, several solutions are available. First, network speed can be increased by upgrading to fiber-distributed data interface (FDDI) or copper-distributed data interface (CDDI); this in- 
creases top network speed from $10 \mathrm{Mbits} / \mathrm{s}$ to $100 \mathrm{Mbits} / \mathrm{s}$. However, implementation of FDDI or CDDI is expensive because it requires recabling (at least for FDDI) and new interface cards. A second solution is to setup a subnet exclusively for nuclear medicine use. This removes traffic from the Ethernet backbone. A third option is to shift as much of the processing to the remote system as possible.

In general, the UNIX workstations meet the requirements for a diagnostic workstation, at least for nuclear medicine. ${ }^{3.15}$ The screen rewrite speed of some of the workstations (IPX and SPARC 2) is slower that the Stardent's video display. This was not a problem for routine image display, but rotation of $3 \mathrm{D}$ reconstructions was significantly slower than on the main system.
In summary, we present a simple and low-cost PACS solution for nuclear medicine that is based on redirection of client/server relationship of the $\mathrm{X}$ Window system. The technique requires that the main computer system's hardware, operating system, and imaging software support multiuser multitasking and the simultaneous execution of multiple copies of its imaging software, and that it use the $\mathrm{X}$ Window system. Advantages of our solution include ease of interconnection of different types of computers, the ability to view images regardless of file format, and the capacity to view and process images on remote systems. A disadvantage is the limited number of nodes that can be supported and still maintain adequate performance.

\section{REFERENCES}

1. Choplin RH, Boehme JM, Maynard CD, et al: PACS mini refresher course: Picture archiving and communication systems: An overview. RadioGraphics 12:127-129, 1992

2. Worrell JA, Federspiel CF, Creasy JL, et al: Clinical impact of picture archiving and communication systems: Evaluation of prototype system. J Digit Imaging 5:118-125, 1992

3. Haynor DR. Smith DV, Park HW, et al: Hardware and software requirements for a picture archiving and communication system's diagnostic workstations. J Digit Imaging 5:107-117, 1992

4. Naylor AF: A strategy for the progressive introduction of picture archiving and communication systems for a radiology department. J Digit Imaging 5:82-88, 1992

5. Staab EV: PACS mini refresher course: Introduction. RadioGraphics 12:125-126, 1992

6. Brown PH, Krishnamurthy GT: Design and operation of a nuclear medicine picture archiving and communication system. Semin Nucl Med 20:205-224, 1990

7. Hindel R: Digital image storage technology. Invest Radiol 28:454-458, 1993

8. Datz FL, Christian PE, Ahluwalia R, et al: Comparison of 3-D surface and transparency renderings to SPECT tomographic slice images for the detection of coronary artery disease. J Nucl Med 34:196, 1993 (abstr)

9. Bedont RT, Taylor AT. Verba J, et al: Application of phase analysis to nuclear cardiology. Appl Radiol 15:49-57, 1986

10. Datz FL, Gabor FV, Christian PE, et al: Use of computer-assisted diagnosis in cardiac perfusion nuclear medicine studies: A review (part 2). J Digit Imaging 6:1-15. 1993

11. Reiss L, Radin J: X Window Inside and Out. Berkley, CA. Osborne McGraw-Hill, 1992

12. Young DA: The $X$ Window System: Programming and Applications with Xt: OSF/Motif Edition. Englewood Cliffs, New Jersey, Prentice-Hall, 1990

13. Huang HK: PACS mini refresher course: Three methods of implementing a picture archiving and communication system. RadioGraphics 12:131-139, 1992

14. Datz FL. Gullberg GT, Zeng GL, et al: Application of convergent-beam collimation and simultaneous transmission emission tomography to cardiac SPECT imaging. Semin Nucl Med 24:17, 1994 .

15. Lee Kai: Computers in Nuclear Medicine: A Practical Approach. New York, NY, Society of Nuclear Medicine. 1991 